\title{
Preparation and Characterization of Poly[(4-vinylphenyl)dimethylvinylsilane] via Anionic Living Polymerization
}

\author{
Kazunori SE, ${ }^{\dagger}$ Kohzaburoh Matsumura, ${ }^{*}$ Takeo KaZama, ${ }^{*}$ \\ and Teruo Fujimoto* \\ Department of Materials Science and Engineering, Faculty of Engineering, Fukui University, \\ Bunkyo 3-9-1, Fukui 910, Japan \\ * Department of Chemistry, Faculty of Engineering, Nagaoka University of Technology, \\ Kamitomioka 1603-1, Nagaoka, Niigata 940-21, Japan
}

(Received October 3, 1996)

\begin{abstract}
Vinylphenyl)dimethylvinylsilane (VS) was anionically polymerized to yield poly[(4-vinylphenyl)dimethylvinylsilane] (PVS). The styryl group of VS was polymerized and silylvinyl group of VS remained unreacted during the anionic polymerization. However, a slight amount of the silylvinyl group of VS should be subject to anionic polymerization. To suppress two side reactions related to the silylvinyl group, the influence of initiators, solvents and polymerization time on anionic polymerization was studied in detail. When $n$-butyllithium was used as initiator, the resultant polymer conversion rate was $60 \%$ and the corresponding GPC chromatogram showed a broad peak. This is because during chain propagation, the styryl living end was deactivated by a labile proton of the silylmethyl group of the side chain (abstraction mechanism). When cumylpotassium and cumylcesium were used as initiators, the polymer conversion rates were nearly $100 \%$ and corresponding GPC chromatograms showed double and/or triple peaks because after chain propagation had completely finished, the styryl living end reacted with the silylvinyl group of the side chain of a different polymer molecule (addition mechanism). When a mixture of diethyl ether/4,4-dimethyl-1,3-dioxane (1,3-DX) was used as solvent, PVS yielded a sharp GPC chromatogram containing a small shoulder at the higher molecular weight side. The size of this shoulder increased with polymerization time. Thus, VS was polymerized by cumylcesium in a $1: 2$ mixture of diethyl ether/1,3-DX for 30 minutes to yield four polymers with $M_{n}$ from $2.5_{0} \times 10^{4}$ to $1.3_{6} \times 10^{5}$, and $M_{w} / M_{n}$ less than $1.0_{8}$. Upon exposure to a deep ultraviolet light having a wavelength of $290 \mathrm{~nm}$, gel of PVS was observed in the exposed area.

KEY WORDS Anionic Living Polymerization / Poly[(4-vinylphenyl)dimethylvinylsilane] / Narrow Molecular Weight Distribution / Cumylcesium / Negative Resist /
\end{abstract}

Polymers that contain a vinyl group as a side chain are interesting stable intermediates of final polymers, because they can be used as backbone chains of graft copolymers,,${ }^{1,2}$ as prepolymers of cross-linked polymers, ${ }^{3}$ and for introducing other functions. ${ }^{4}$ The polymers themselves form intermolecular cross-linkages, and thus can be used as negative working resists. ${ }^{5-7}$ Polymers as intermediates of the final high-performance polymers must have a narrow molecular weight distribution (MWD). ${ }^{5,8-10}$

Thus, a new monomer, (4-vinylphenyl)dimethylvinylsilane (VS) was designed by the present authors. ${ }^{1,11}$ As shown in Figure 1, VS contains two vinyl groups of a styryl group and a silylvinyl group. Although the silylvinyl group of VS is thought much less reactive than the styryl group of VS under anionic condition, both vinyl groups are capable of polymerizing anionically in a polar solvent. When VS reacted with cumyl-potassium in THF under high vacuum, the styryl group was polymerized to give the polymer of molecular weight less than $10^{4}{ }^{1,12}$ To prepare a well-defined poly[(4-vinylphenyl)dimethylvinylsilane] (PVS) of a molecular weight much larger than $10^{4}$, a systematic experiment of anionic polymerization was carried out in this study.

The first object of this study was to suppress the polymerization of the silylvinyl group of VS and to carry out selective polymerization of the styryl group of VS. The second object was to suppress the side reaction relating to the silylvinyl group during the process of chain propagation of the styryl group, and prepare a well-defined PVS of high molecular weight and narrow MWD. For this purpose, the influence of initiators, solvents, and polymerization time on anionic polymerization was studied in detail.

This polymer contains a silicon atom. ${ }^{13,14}$ Siliconbased resists ${ }^{15}$ can be applied to multi-layer resists ${ }^{16,17}$ and to practical submicron lithography due to planning of the fabricated substrate surface. ${ }^{5,6} \mathrm{~A}$ series of preliminary experiments concerning lithographic evaluation was carried out.

\section{EXPERIMENTAL}

\section{Reagents}

(4-Vinylphenyl)dimethylvinylsilane (VS) was prepared as follows: $p$-chlorostyrene $(65 \mathrm{~g})$ reacted with magnesium turnings $(12 \mathrm{~g})$ in THF $(200 \mathrm{ml})$ containing ethylbromide<smiles>C=Cc1ccc([Si](C)(C)C=C)cc1</smiles>

Figure 1. Structural formula of (4-vinylphenyl)dimethylvinylsilane (VS) containing a styryl group and silylvinyl group.

† To whom all correspondence should be addressed (Tel: +81-776-27-8957, Fax: +81-776-27-8767). 
$(0.5 \mathrm{ml})$ to yield a Grignard reagent. Vinyldimethylchlorosilane $(60 \mathrm{~g})$ was added to the Grignard reagent at room temperature. Following hydrolysis of the product, VS $(75 \mathrm{~g})$ was prepared at $80 \%$ yield. The VS was distilled twice at $74^{\circ} \mathrm{C} / 2 \mathrm{mmHg}$. Purity of the VS was checked by gas chromatography and ${ }^{1} \mathrm{H}$ NMR. Subsequent purification of VS was carried out at $10^{-5}$ Torr in an allglass apparatus equipped with breakseals. After being dried with calcium hydride, VS was further purified twice with octylbenzophenone sodium anion as previously done. ${ }^{18-20}$ VS was diluted with carefully purified solvents for anionic polymerization.

n-Butyllithium (n-BuLi) was diluted with purified $n$-heptane. Cumylpotassium (Cumyl K) was synthesized via reaction of cumylmethyl ether with sodium-potassium alloy in THF, and cumylcesium (Cumyl Cs) was synthesized via reaction of cumylmethyl ether with cesium in THF. Concentrations of the initiators were determined by titration with standard hydrochloric acid.

Diethyl ether (Ether), 2-methyltetrahydrofuran (MeTHF), THF, and 4,4-dimethyl-1,3-dioxane (1,3-DX) were used as solvents for the polymerization. The former three solvents are commercial products and 1,3-DX was supplied by Fine Chemicals Department, Mitsubishi Chemical Corporation. They were dried with sodiumwire and distilled in vacuum with anthracene sodium anion. Finally, they were distilled over $\alpha$-methylstyrene tetramer-sodium dianions. After conventional purification, $n$-heptane for dilution of the initiator was distilled over $n$-BuLi.

\section{Polymerization}

Polymerization was carried out at $-78^{\circ} \mathrm{C}$ at $10^{-5}$ Torr for $0.5-3 \mathrm{~h}$. The polymerization techniques were almost the same as those in previous studies. ${ }^{18-20}$ The polymerization was terminated by methanol and PVS was separated as a precipitate by introducing the polymer solution to excess methanol. The precipitate was carefully washed with fresh methanol and dried under high vacuum.

\section{Molecular Characterization}

${ }^{1} \mathrm{H}$ NMR spectra of VS and the polymer were measured in $\mathrm{CDCl}_{3}$ containing tetramethylsilane at room temperature using JNM-GX270 FT NMR spectrometer (JEOL Ltd.). IR spectra of VS and the polymer were recorded on a JASCO IRA-102 spectrophotometer (Japan Spectroscopic Co., Ltd.). All polymer samples were tested by gel-permeation chromatography (GPC, Model $801 \mathrm{~A}$, Tosoh Co.) with two high resolution columns of G-4000H8 $(7.8 \mathrm{~mm}$ i.d. $\times 60 \mathrm{~cm}$, Tosoh Co.) or two high resolution columns of G-5000H6 $(7.8 \mathrm{~mm}$ i.d. $\times 60 \mathrm{~cm}$, Tosoh Co.) using standard polystyrenes (TSK polystyrenes, Tosoh Co.) to estimate molecular weight heterogeneities $\left(M_{w} / M_{n}\right.$ values). THF was used as carrier solvent. Number average molecular weights, $M_{n}$ of several PVS samples were also determined by osmometry in toluene at $30^{\circ} \mathrm{C}$ using a Hewlett-Packard Type 502 high-speed membrane osmometer. Differential scanning calorimetry was carried out using DSC (Rigaku Denki Co., CN-8059-A2 Type) at a heating rate of $10 \mathrm{~K} \mathrm{~min}^{-1}$

\section{RESULTS AND DISCUSSION}

Preparation of Poly[(4-vinylphenyl)dimethylvinylsilane].

When VS was polymerized by cumylpotassium (Cumyl $\mathrm{K}$ ) in $\mathrm{THF}$ at $-50^{\circ} \mathrm{C}$ under nitrogen atmosphere as a preliminary experiment, ${ }^{10}$ the polymerization solution became viscous and a gel-like product was obtained. Although the silylvinyl group of VS was much less reactive than the styryl group of VS in a polar solvent, ${ }^{12}$ both vinyl groups were simultaneously polymerized under the preliminary experimental condition. When anionic polymerization of VS was carried out at $-78^{\circ} \mathrm{C}$ under high vacuum for less than $3 \mathrm{~h}$ using break-seal technique, a gel-like product was not obtained. A linear polymer without intermolecular crosslinkages should be produced by anionic polymerization. The first object of this study is to determine which vinyl group of VS was subject to the anionic polymerization. Spectroscopic characterization was carried out for this purpose.

${ }^{1} \mathrm{H}$ NMR spectrum of VS is shown in Figure 2. Taking into account the chemical shifts of methine proton $(6.73-6.77 \mathrm{ppm})$ and methylene protons (5.24-5.29 ppm and $5.81-5.88 \mathrm{ppm})$ of the vinyl group of 4vinylbenzyl chloride $e^{21-23}$ as a reference sample, b signals $(6.68-6.75 \mathrm{ppm})$ in Figure 2 and a signals $(5.23-5.29 \mathrm{ppm}$ and $5.78-5.87 \mathrm{ppm})$ in Figure 2 were assigned to the methine proton and methylene protons of the styryl group of VS. The methine proton of the silylvinyl group should be observed at the lower ppm than the corresponding proton of the styryl group due to difference in electron releasing ${ }^{24}$ between silicon atom and phenyl group. Hence, e signals $(6.20-6.35 \mathrm{ppm})$ in Figure 2 were assigned to the methine proton of the silylvinyl group of VS. ${ }^{14,24}$ The methylene protons of the silylvinyl group should be observed at the higher ppm than the corresponding protons of the styryl group due to no resonance structure in the silylvinyl group, compared with the resonance structure of the styryl group. Hence, f signals (5.68-5.72 ppm and 6.05-6.10 ppm) in Figure 2 were assigned to the methylene proton of the silylvinyl group of VS. ${ }^{14,24}$ Assignment of all NMR

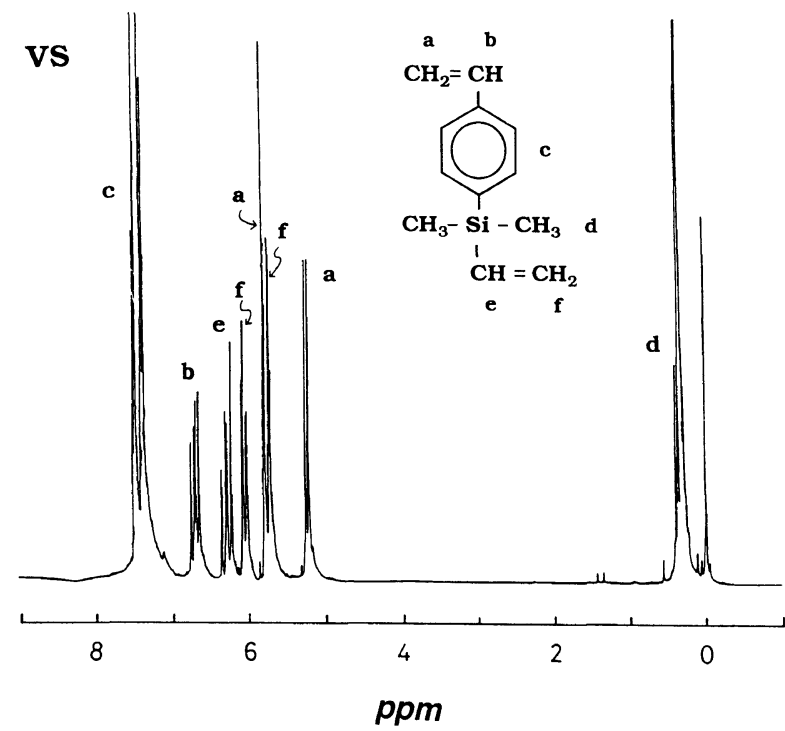

Figure 2. $270 \mathrm{MHz}{ }^{1} \mathrm{H}$ NMR spectrum of (4-vinylphenyl)dimethylvinylsilane (VS) in $\mathrm{CDCl}_{3}$ containing tetramethylsilane. Assignments of all NMR signals are shown in the figure. 


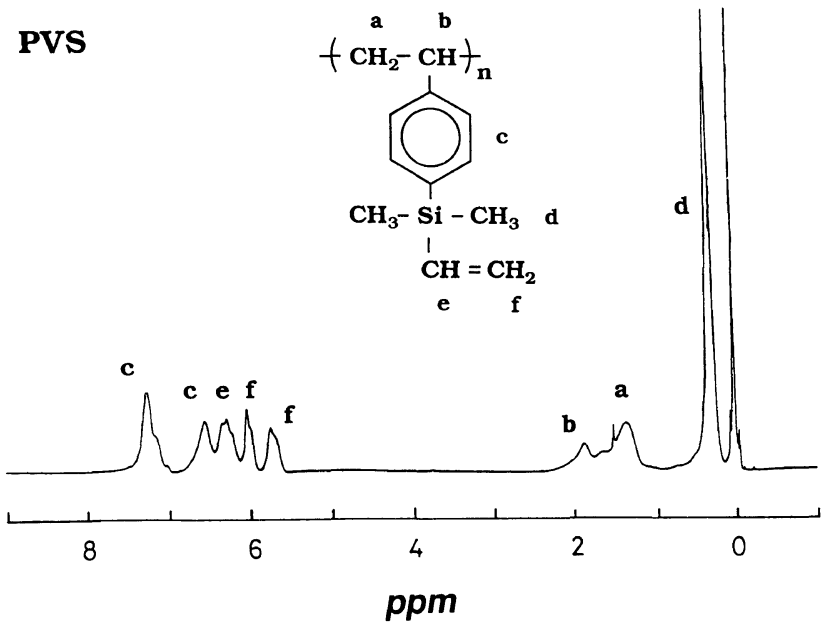

Figure 3. $270 \mathrm{MHz}{ }^{1} \mathrm{H}$ NMR spectrum of poly[(4-vinylphenyl)dimethyl-vinylsilane] (PVS) in $\mathrm{CDCl}_{3}$ containing tetramethylsilane. Assignments of all NMR signals are shown.

signals of VS is also shown in Figure 2. The intensity of the resultant NMR signals agreed quantita tively with the structure of VS.

The difference in chemical shifts of the vinyl groups between 4-vinylbenzyl chloride and (4-vinylbenzyl)polystyrene macromonomer was less than $0.1 \mathrm{ppm} .{ }^{21-23}$ That is, the chemical shift of the vinyl group was scarcely affected whether a polymer chain attached to the styryl group or not. This should help to assign the NMR spectrum of the resultant polymer. Chemical shifts of e and $f$ signals of the resultant polymer in Figure 3 exactly coincided with those of $e$ and f signals of VS in Figure 2. $a$ and $b$ signals of VS completely disappeared in the NMR spectrum of the resultant polymer. The styryl group of VS is thus chemoselectively polymerized by the anionic mechanism to give the polymer. Assignment of all NMR signals of PVS is also shown in Figure 3. The intensity of the resultant NMR signals agreed quantitatively with the structure of PVS.

The same conclusion was made for the results of IR spectra of VS and resultant polymer. Special attention should be directed to the two strong absorption bands at $1600 \mathrm{~cm}^{-1}$ and $1640 \mathrm{~cm}^{-1}$ shown in Figure 4. Polystyrene showed a weak absorption band at $1600 \mathrm{~cm}^{-1}$ and medium absorption bands at $1440 \mathrm{~cm}^{-1}$ and 1480 $\mathrm{cm}^{-1}$, which were assigned to in-plane deformation vibration of the benzene ring. ${ }^{14,24}$ As shown in Figure 4 , VS showed the corresponding medium absorption bands at $1516 \mathrm{~cm}^{-1}\left(1502 \mathrm{~cm}^{-1}\right)$ and $1558 \mathrm{~cm}^{-1}$. However, the observed absorption at $1600 \mathrm{~cm}^{-1}$ was much larger than the expected absorption of the benzene ring. Hence, most of the strong absorption bands at 1600 $\mathrm{cm}^{-1}$ were due to the vinyl $\mathrm{C}=\mathrm{C}$ stretching vibration of VS. ${ }^{14,24}$ The stretching energy was calculated from the simple harmonic vibration approximation as follows ${ }^{24}$;

$$
v\left(\mathrm{~cm}^{-1}\right)=4.12\left(10^{6} / \mu\right)^{1 / 2}
$$

where $\mu$ is reduced weight of a vinyl group of $M_{1}=M_{2}$; namely $\mu=M_{1} M_{2} /\left(M_{1}+M_{2}\right) . M_{1}$ and $M_{2}$ are reduced atomic weights of both atoms in the vinyl group. For a simple example of $\mathrm{C}=\mathrm{C}, M_{1}$ and $M_{2}$ are 6. And $\mu=6$ and $v=1682 \mathrm{~cm}^{-1} . \mu$ of the styryl group $\left(\mathrm{CH}_{2}=\mathrm{CH}-\mathrm{C}\right)$

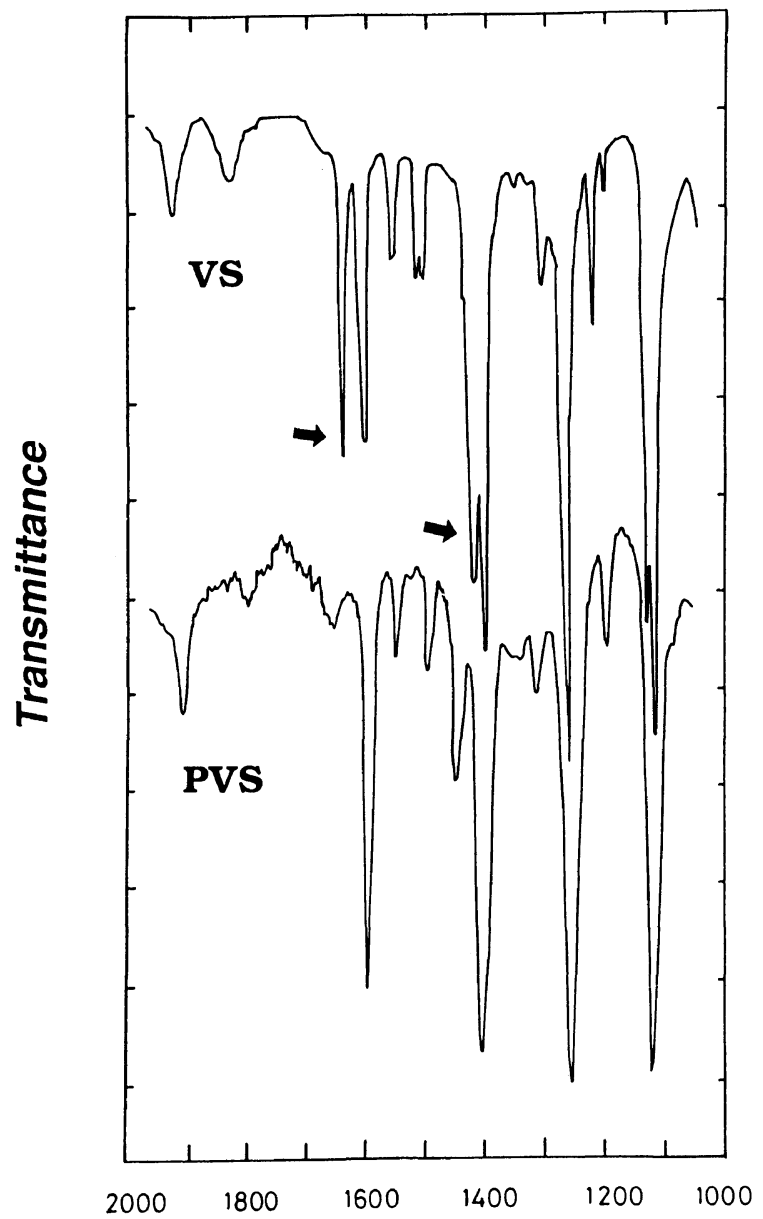

Wave Number $/ \mathrm{cm}^{-1}$

Figure 4. Infrared spectra of (4-vinylphenyl)dimethylvinylsilane (VS) and poly[(4-vinylphenyl)dimethylvinylsilane] (PVS) in the region between $1000-2000 \mathrm{~cm}^{-1}$. Two arrows show the absorption bands of the vinyl $\mathrm{C}=\mathrm{C}$ stretching vibration of the styryl group of VS.

is thought to be smaller than that of the silylvinyl group $\left(\mathrm{CH}_{2}=\mathrm{CH}-\mathrm{Si}\right)$ because of the difference in atomic weight between the carbon and silicon. When $\mu$ is 6.31, $v=$ $1640 \mathrm{~cm}^{-1}$ and when $\mu$ is $6.63, v=1600 \mathrm{~cm}^{-1}$. Although this could be derived from approximate calculation, ${ }^{24}$ it is worth noting that the absorption band of the styryl group of VS should appear at the higher energy region than that of the silylvinyl group of VS. The observed absorption band at $1640 \mathrm{~cm}^{-1}$ in Figure 4 and that at $1600 \mathrm{~cm}^{-1}$ in Figure 4 were thus assigned to the $\mathrm{C}=\mathrm{C}$ stretching vibrations of the styryl group and silylvinyl group of VS, respectively.

On comparing the two IR spectra of VS and the resultant polymer, the absorption band of the styryl group and that of the silylvinyl group of VS, were respectively found to disappear and remain in the IR spectrum of the resultant polymer. The same was observed in the absorption bands of VS and the resultant polymer at around $1400 \mathrm{~cm}^{-1}$ assigned to the $\mathrm{C}=\mathrm{C}$ bending of the styryl group and silylvinyl group. The styryl group of VS is thus selectively polymerized under the anionic condition to yield poly[(4-vinylphenyl)dimethylvinylsilane] (PVS). 
Table I. Anionic polymerization of (4-vinylphenyl)dimethylvinylsilane (VS) with various initiators

\begin{tabular}{|c|c|c|c|c|c|c|}
\hline \multirow{4}{*}{ Solvent } & \multicolumn{2}{|c|}{ Initiator } & \multirow{4}{*}{$\frac{\text { Monomer }}{\text { mol 1 }^{-1}}$} & \multirow{4}{*}{$\frac{\text { Time }}{h}$} & \multirow{4}{*}{$10^{-4} M_{k}^{\mathrm{a}}$} & \multirow{4}{*}{$\frac{\text { Conversion }}{\%}$} \\
\hline & \multirow{3}{*}{ Name } & \multirow{3}{*}{$\frac{10^{4}[\mathrm{I}]}{\mathrm{mol1}^{-1}}$} & & & & \\
\hline & & & & & & \\
\hline & & & & & & \\
\hline THF & $n$-BuLi & $16 \cdot 3$ & $0.43_{5}$ & 3.0 & $3.0_{1}$ & 60 \\
\hline $\mathrm{THF}$ & Cumyl K & 19.6 & $0.31_{5}$ & 3.0 & $3.0_{2}$ & 100 \\
\hline THF & Cumyl Cs & 20.4 & $0.43_{5}$ & 3.0 & $4.0_{1}$ & 100 \\
\hline
\end{tabular}

a Molecular weights calculated from amounts of monomer and initiator.

\section{Experimental Factors of Anionic Polymerization}

When a new monomer is polymerized by anionic polymerization to yield a so-called monodispersed polymer, particular attention should be directed to initiator, solvent, polymerization time, polymerization temperature, concentration of initiator, and concentration of monomer. The first four factors are more influential than the latter two. Although the detailed mechanism is not known, a lower temperature is a favorable to suppress the gel-like product. Hence, in the present study polymerization temperature was set at $-78^{\circ} \mathrm{C}$, concentration of the initiator approximately $10^{-3} \mathrm{moll}^{-1}$, and concentration of the monomer approximately $2 \times 10^{-1} \mathrm{moll}^{-1}$. A kinetic molecular weight $M_{k}$, calculated from the amounts of monomer and initiator used in the polymerization, fell within the range of $3 \times 10^{4}-4 \times 10^{4}$. Initiator, solvent, and polymerization time were investigated in detail to find means for suppressing side reactions in the polymerization and prepare PVS of narrow MWD.

\section{Initiators}

The styryl group of VS was first anionically polymerized using THF as solvent, $3 \mathrm{~h}$ as polymerization time, and $n$-BuLi, Cumyl K, and Cumyl Cs as initiators. The polymerization results and GPC chromatograms are shown in Table I and Figure 5, respectively. When $n$-BuLi was used as initiator, the polymer conversion rate was $60 \%$ and the corresponding GPC chromatogram showed a single but broad peak. Based on a comparison with other polymer conversion rates of $100 \%$ using Cumyl K and Cumyl $\mathrm{Cs}$ as initiators for $3 \mathrm{~h}$, the polymer conversion rate of $60 \%$ for $3 \mathrm{~h}$ should not increase even though for longer polymerization time. These results suggest that the living carbanion of the styryl group is deactivated step by step before chain propagation completely finishes. Taking into account the polymer conversion rates of $100 \%$ using Cumyl $\mathrm{K}$ and Cumyl Cs, this deactivation is thought to be attributed not to impurities present in the reaction system but to a specific mechanism of this polymerization system.

Two termination mechanisms of the styryl living end are presumed to operate in the polymerization. The first termination mechanism concerns addition of the styryl living end to the silylvinyl group of the side chain. When this termination occurred in the same polymer molecule, the molecular weight of the resultant PVS corresponded to the value of $M_{k}$, as is the case of disproportionation. When this termination occurred in the different polymer molecules, the molecular weight of the resultant PVS corresponded two to three times the value of $M_{k}$, as is

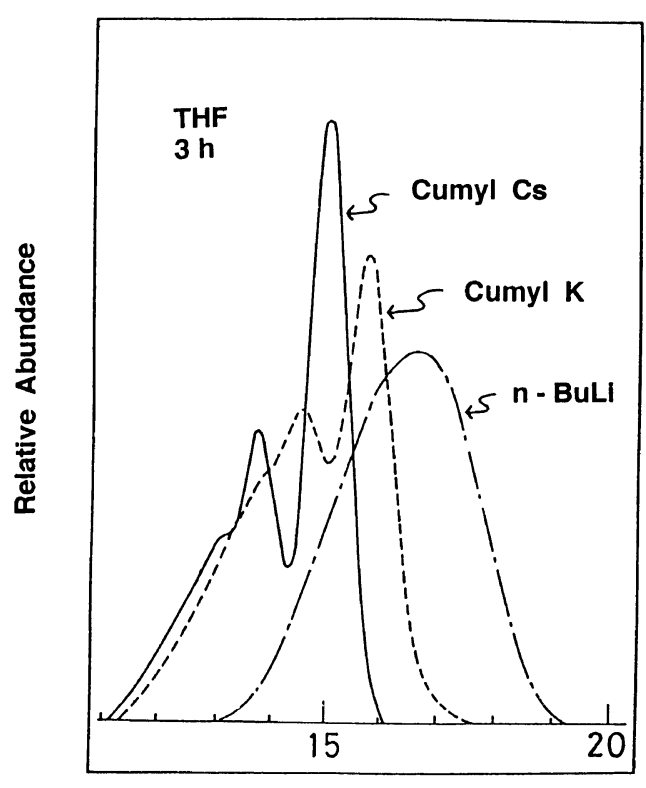

Elution Volume / ml

Figure 5. GPC chromatograms of PVS prepared using $n$-BuLi, Cumyl $\mathrm{K}$, and Cumyl Cs as initiators, THF as solvent, and polymerization time of $3 \mathrm{~h}$. Two high resolution columns of $\mathrm{G}-4000 \mathrm{H} 8$ were used for the measurement.

the case of a combination reaction or a coupling reaction. In contrast, the second termination mechanism is abstraction of a labile proton by the styryl living end, from the methyl group on the silicon atom. When this termination of the styryl living ends occurs in the same polymer molecule and/or the different polymer molecules, the molecular weight of the resultant PVS corresponds to the value of $M_{k}$, as is the case of disproportionation.

When the styryl group of VS was polymerized by $n$ $\mathrm{BuLi}$, most of the deactivation of the styryl living end was presumed to occur by the second termination mechanism (abstraction mechanism). This termination was found to occur not after chain propagation was complete but during chain propagation, as the polymer conversion rate was not $100 \%$ and GPC chromatogram of PVS showed one broad peak.

When Cumyl $\mathrm{K}$ and Cumyl Cs were used as initiators, the polymer conversion rates were nearly $100 \%$. The corresponding GPC chromatograms show sharp double and/or triple peaks. $M_{n}$ of each peak from a lower molecular weight side of double and/or triple peaks was found to correspond to $M_{k}, 2 M_{k}$, or $3 M_{k}$. After chain propagation completely finishes, the styryl living end may thus become attached to the silylvinyl group of the side 
K. SE et al.

Table II. Anionic polymerization of (4-vinylphenyl)dimethylvinylsilane (VS) in various solvents

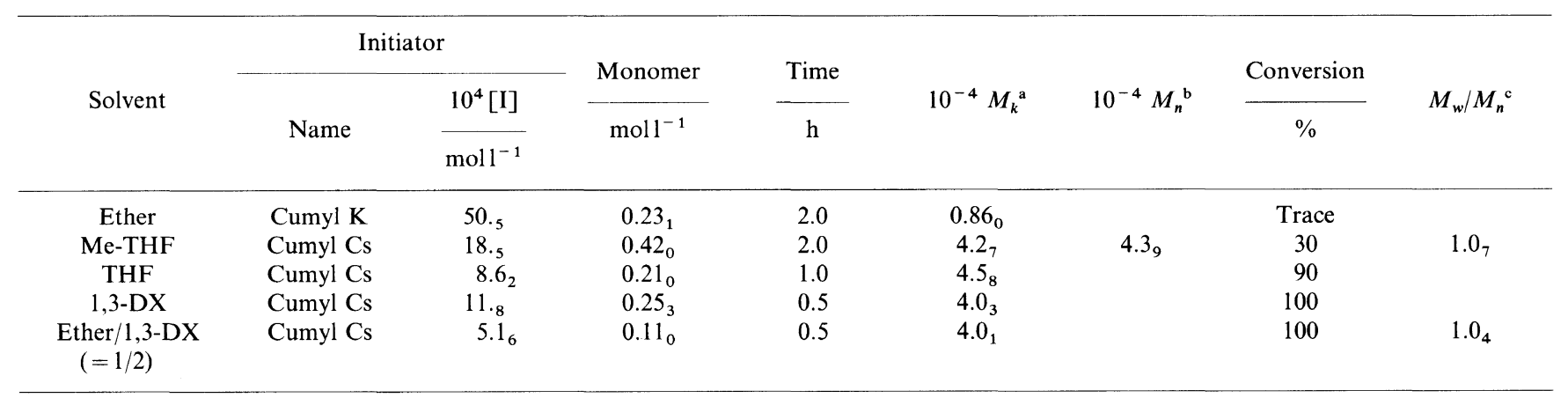

a Molecular weights calculated from amounts of monomer and initiator. b Number average molecular weights determined by membrane osmometry. ${ }^{\mathrm{c}}$ Molecular heterogeneity determined by GPC measurement.

chain of another polymer molecule. That is, most of the polymerization was terminated not by the second mechanism (abstraction mechanism) but by the first mechanism (addition mechanism). An intermolecular combination of different polymer molecules resulted in the formation of a dimer and trimer of the primary living molecules.

During anionic polymerization of the styryl group of VS, propagation is believed to be in competition with the two side reactions. Cumyl $\mathrm{K}$ and Cumyl Cs appear to be favorable initiators of chain propagation for preparing PVS with narrow MWD.

\section{Solvents}

VS was polymerized by Cumyl $\mathrm{K}$ and Cumyl Cs using $0.5-2 \mathrm{~h}$ as polymerization time in diethyl ether (Ether, dielectric constant, $\varepsilon$ of 4.4 at $17^{\circ} \mathrm{C}$ ), 2-methyltetrahydrofurane (Me-THF, $\varepsilon$ of 6.3 at $18^{\circ} \mathrm{C}$ ), ${ }^{23}$ THF ( $\varepsilon$ of 8.2, at $20^{\circ} \mathrm{C}$ ), and 4,4-dimethyl-1,3-dioxane (1,3-DX, $\varepsilon$ of 7.34 at $25^{\circ} \mathrm{C}$ ) as solvents. The polymerization results are shown in Table II. When Ether and Me-THF were used as solvents for anionic polymerization, the polymer conversion rates were approximately $0 \%$ and $30 \%$, respectively. As shown in Figure 6, a GPC chromatogram of PVS prepared using Me-THF shows one peak, which has a shoulder at the higher molecular weight side.

When THF was used as solvent, PVS was prepared at a $90 \%$ conversion rate. Its chromatogram showed two peaks. The respective $M_{n}$ of the lower molecular weight peak and higher molecular weight peak correspond to $M_{k}$ and $2 M_{k}$, respectively. Taking into account the polymer conversion rate of $90 \%$, the first termination mechanism of the styryl living end (addition mechanism) was thought to occur after chain propagation nearly finished.

When 1,3-DX was used as solvent, PVS was prepared at a conversion rate of $100 \%$, and its chromatogram showed two peaks. $M_{n}$ of the two peaks were found to be much larger than the $M_{k}$ value and $2 M_{k}$ value, respectively. The freezing point of $1,3-\mathrm{DX}$ is $-88.5^{\circ} \mathrm{C}$; hence this solvent became viscous at $-78^{\circ} \mathrm{C}$, at which temperature VS was polymerized. When 1,3-DX solution of VS was added to this viscous solution of the initiator, VS could not be polymerized homogeneously but was polymerized on the interface between the initiator solution and monomer solution to yield PVS that had higher $M_{n}$. In spite of this, VS was polymerized for 30 minutes

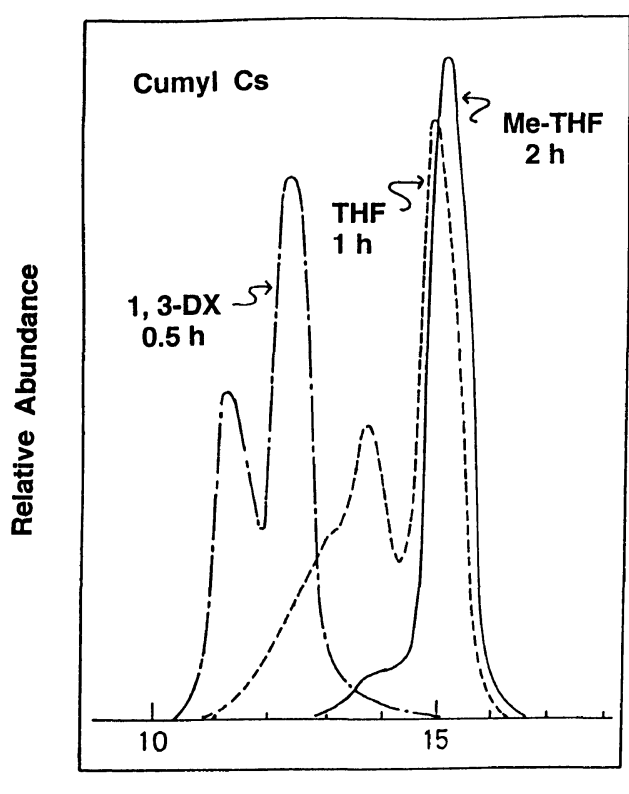

Elution Volume / $\mathrm{ml}$

Figure 6. GPC chromatograms of PVS prepared using tetrahydrofuran (THF), Me-THF, and 1,3-DX as solvents, Cumyl Cs as an initiator, and polymerization time as described in figure. Two high resolution columns of $\mathrm{G}-4000 \mathrm{H} 8$ were used for the measurement.

with a conversion rate of $100 \%$. These results suggest that the polymerization rate in $1,3-\mathrm{DX}$ is considerably faster than that in Me-THF and THF.

To reduce the high viscosity of $1,3-\mathrm{DX}$ as a solvent and carry out homogeneous polymerization at $-78^{\circ} \mathrm{C}$, a mixed solvent of Ether and 1,3-DX was used as solvent. With the addition of Ether to 1,3-DX, the viscosity of the mixed solvent decreases and the mixed solvent should retain high polarity. The polymerization results are shown in Table II and Figure 7. Polymerization was performed homogeneously in a $1: 2$ mixture of Ether/ $1,3-\mathrm{DX}$ (volume ratio). After 30 minutes, the polymer conversion rate was $100 \%$ and the corresponding GPC chromatogram of PVS showed a sharp peak containing a small shoulder at the higher molecular weight side. That is, most of the side reactions of the styryl living end relating to the dimethylvinylsilyl group could be suppressed. Thus, a mixed solvent of Ether/1,3-DX is useful for preparing well-defined PVS of high molecular weight and narrow MWD. 

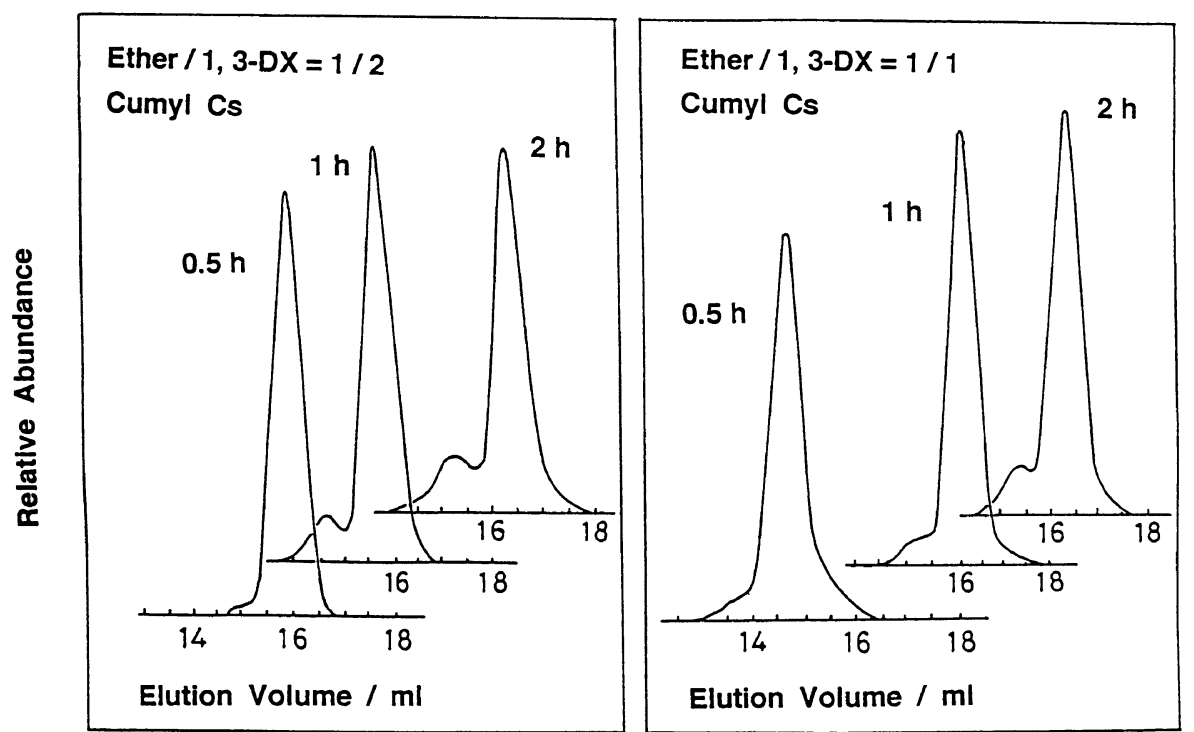

Figure 7. GPC chromatograms of PVS prepared at polymerization time between $0.5 \mathrm{~h}$ and $2 \mathrm{~h}$, Cumyl Cs as initiator, and mixture of Ether/1,3-DX =1/2 (a left figure) and Ether/1,3-DX =1/1 (a right figure). Two high resolution columns of G-5000H6 were used for measurement of the sample prepared in Ether/1,3-DX $=1 / 2$ for $0.5 \mathrm{~h}$. The five other samples were eluted by two high resolution columns of G-4000H8.

Table III. Anionic polymerization of (4-vinylphenyl)dimethylvinylsilane (VS) in a mixture of Ether/1,3-DX

\begin{tabular}{|c|c|c|c|c|c|c|c|}
\hline \multirow{3}{*}{ Solvent } & \multicolumn{2}{|c|}{ Initiator } & \multirow{3}{*}{$\frac{\text { Monomer }}{\mathrm{moll}^{-1}}$} & \multirow{3}{*}{$\frac{\text { Time }}{\mathrm{h}}$} & \multirow{3}{*}{$10^{-4} M_{k}^{\mathrm{a}}$} & \multirow{3}{*}{$\frac{\text { Conversion }}{\%}$} & \multirow{3}{*}{$M_{w} / M_{n}^{\mathrm{b}}$} \\
\hline & \multirow{2}{*}{ Name } & \multirow{2}{*}{$\frac{10^{4}[\mathrm{I}]}{\mathrm{moll}^{-1}}$} & & & & & \\
\hline & & & & & & & \\
\hline Ether $/ 1,3-\mathrm{DX}=1 / 2$ & Cumyl Cs & $2.3_{2}$ & $0.12_{9}$ & 0.5 & 10.5 & 100 & $1.0_{3}$ \\
\hline Ether $/ 1,3-\mathrm{DX}=1 / 2$ & Cumyl Cs & $5.3_{5}$ & $0.11_{0}$ & 1.0 & $3.8_{7}$ & 100 & $1.0_{7}$ \\
\hline Ether $/ 1,3-\mathrm{DX}=1 / 2$ & Cumyl Cs & $5.5_{9}$ & $0.10_{8}$ & 2.0 & $3.6_{3}$ & 100 & $1.1_{1}$ \\
\hline Ether/1,3-DX $=1 / 1$ & Cumyl Cs & $2.9_{2}$ & $0.09_{8}$ & 0.5 & $6.3_{1}$ & 100 & $1.0_{4}$ \\
\hline Ether/1,3-DX $=1 / 1$ & Cumyl Cs & $7.2_{6}$ & $0.11_{4}$ & 1.0 & $2.9_{5}$ & 100 & $1.0_{5}$ \\
\hline Ether $/ 1,3-\mathrm{DX}=1 / 1$ & Cumyl Cs & $6.7_{6}$ & $0.12_{8}$ & 2.0 & $3.5_{6}$ & 100 & $1.0_{9}$ \\
\hline
\end{tabular}

${ }^{a}$ Molecular weights calculated from amounts of monomer and initiator. ${ }^{\mathrm{b}}$ Molecular heterogeneity determined by GPC measurement.

\section{Polymerization Time}

Cumyl Cs was used as an initiator and a $1: 2$ or $1: 1$ mixture of Ether/1,3-DX (volume fractions) as the solvent. To prepare PVS with narrow MWD, the side reaction caused by the first mechanism (addition mechanism) must be suppressed. VS was polymerized, using polymerization times of 30 minutes, $1 \mathrm{~h}$, and $2 \mathrm{~h}$. The polymerization results are shown in Table III and Figure 7 . In all cases, the polymer conversion rates were $100 \%$. The GPC chromatogram of each PVS shows one sharp peak having a small shoulder at the higher molecular weight side, and the size of the shoulder was found to increase with polymerization time. Addition of the styryl living end to the silylvinyl group of other polymer molecules was believed to occur after chain propagation completely finished. This would account for the increase in the size of the shoulder. The molecular heterogeneity or $M_{w} / M_{n}$ of each GPC main peak increased with polymerization time from $1.0_{3}$ (30 minutes) to $1.1_{1}(2 \mathrm{~h})$. This is because of another addition of the styryl living end to the silylvinyl group of the same polymer molecule. Ring formation might occur, and its extent increase slightly with time, even though the details are not well known.

These results indicate that the propagation of VS can completely finish in 30 minutes, and subsequent deac- tivation of the styryl living end can occur, thereby yielding a by-product with a molecular weight of $2 M_{k}$ or $3 M_{k}$. Hence, the styryl living end has to be terminated as soon as possible after chain propagation completely finishes. As shown in Table III, the $M_{w} / M_{n}$ of PVS prepared in a 1:2 mixture of Ether/1,3-DX are less than $1.1_{1}$ as also noted for $M_{w} / M_{n}$ of PVS prepared in a $1: 1$ mixture of Ether/1,3-DX.

Thus, we found optimum conditions for preparing a so-called monodispersed PVS by suppressing the side reactions due to the two termination mechanisms. Four PVS samples were prepared by the optimum condition. As shown in Table IV, the polymer conversion rates of the four samples are $100 \%$ and the $M_{n}$ for each of the four samples is good agreement with the corresponding $M_{k}$. We succeeded in preparing well-defined PVS that have $M_{n}$ between $2.5_{0} \times 10^{4}$ and $1.3_{6} \times 10^{5}$, and $M_{w} / M_{n}$ less than $1.0_{8}$.

\section{Lithographic Evaluation of $P V S$}

Differential scanning calorimetry (DSC) of PVS was carried out. The thermograph, covering the temperature range of $290 \mathrm{~K}$ to $450 \mathrm{~K}$, showed an endothermic shift in the vicinity of $303 \mathrm{~K}$. PVS was found to be not a semicrystalline but amorphous polymer, and the glass transition temperature, $T_{\mathrm{g}}$, of PVS was approximately 
K. SE et al.

Table IV. Molecular characteristics of poly[(4-vinylphenyl)dimethylvinylsilane] (PVS) prepared under optimum conditions ${ }^{\mathrm{a}}$

\begin{tabular}{|c|c|c|c|c|c|c|}
\hline \multirow{2}{*}{ Sample } & \multirow{2}{*}{$\frac{\text { Monomer }}{\mathrm{g}}$} & \multirow{2}{*}{$\frac{\text { Initiator }}{10^{4} \mathrm{~mol}}$} & \multirow{2}{*}{$10^{-4} M_{k}^{\mathrm{b}}$} & \multirow{2}{*}{$\frac{\text { Conversion }}{\%}$} & \multirow{2}{*}{$10^{-4} M_{n}^{\mathrm{c}}$} & \multirow{2}{*}{$M_{w} / M_{n}{ }^{\mathrm{d}}$} \\
\hline & & & & & & \\
\hline PVS-1 & $9.5_{3}$ & $3.9_{2}$ & $2.4_{3}$ & 100 & $2.5_{0}$ & $1.0_{5}$ \\
\hline PVS-2 & $8.9_{9}$ & $2.0_{6}$ & $4.3_{6}$ & 100 & $4.5_{9}$ & $1.0_{8}$ \\
\hline PVS-3 & $8.3_{7}$ & $1.8_{1}$ & $4.6_{2}$ & 100 & $4.8_{1}$ & $1.0_{8}$ \\
\hline PVS-4 & $7.4_{5}$ & $0.57_{8}$ & 12.9 & 100 & 13.6 & $1.0_{3}$ \\
\hline
\end{tabular}

a Anionic polymerization using Cumyl Cs as an initiator in a 1:2 mixture of Ether/1,3-DX as a solvent for 30 min at $-78^{\circ} \mathrm{C}$. ${ }^{\mathrm{b}} \mathrm{Molecular}$ weights calculated from amounts of monomer and initiator. ${ }^{c}$ Number average molecular weights determined by membrane osmometry. ${ }^{\mathrm{d}}$ Molecular heterogeneity determined by GPC measurement using standard polystyrenes.

$186 \mathrm{~mJ} / \mathrm{cm}^{2}$ ( 255seconds)

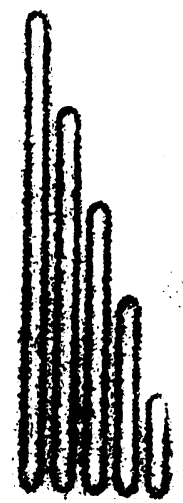

$2.0 \mu \mathrm{m}$
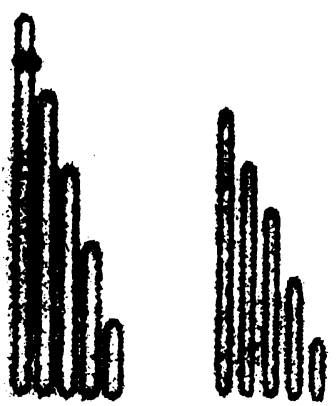

$1.5 \mu \mathrm{m}$

$1.0 \mu \mathrm{m}$
$10 \mu \mathrm{m}$

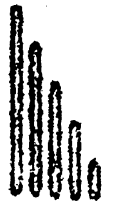

$0.5 \mu \mathrm{m}$
Figure 8. Scanning electron micrographs of image lines from resists of PVS-4 film exposed to deep UV light of $290 \mathrm{~nm}$. A number such as $2.0 \mu \mathrm{m}$ appearing in this figure represents $2.0 \mu \mathrm{m}$ lines and $0.75 \mu \mathrm{m}$ spaces. Intensity of a deep UV light is $186 \mathrm{~mJ} \mathrm{~cm}^{-2}$ (255 seconds).

$303 \mathrm{~K}$, which should be higher than room temperature. Thus, PVS is easier to handle when carrying out lithographic evaluation compared to ordinary cyclized polyisoprene negative resists. ${ }^{25-27}$ PVS is soluble in xylene, toluene, THF, diethyl ether, and other solvents. An ascast film is easily formed from xylene solution. In contrast, PVS is insoluble in alcohols and hexane.

The film of PVS-4 sample was deposited onto a silicon wafer by spin-coating a xylene $(20 \mathrm{wt} \%)$ polymer solution at $500 \mathrm{rpm}$ for 30 seconds. The thickness of the resultant film was approximately $0.5 \mu \mathrm{m}$. The film was exposed to deep UV light with wavelength of $290 \mathrm{~nm}$ and intensity of $186 \mathrm{~mJ} \mathrm{~cm}^{-2}$ (255 seconds) using a Cannon PLA-502F type. The exposed wafers were dip-developed in a mixture of xylene/methanol $=8 / 1$ for 30 seconds and subsequently rinsed with ethanol for 1 minute. Finally, they were postbaked at $60^{\circ} \mathrm{C}$ for 30 minutes. Figure 8 shows scanning electron micrographs of line and space test patterns which have $2.0 \mu \mathrm{m}$ lines and $0.75 \mu \mathrm{m}$ spaces (abbreviated as $2.0 \mu \mathrm{m}$ in the figure), $1.5 \mu \mathrm{m}$ lines and $0.75 \mu \mathrm{m}$ spaces $(1.5 \mu \mathrm{m}), 1.0 \mu \mathrm{m}$ lines and $0.75 \mu \mathrm{m}$ spaces $(1.0 \mu \mathrm{m})$, and $0.50 \mu \mathrm{m}$ lines and $0.75 \mu \mathrm{m}$ spaces $(0.5 \mu \mathrm{m})$ using OAI-205 Type (Optical Associate Inc.). Gels were observed in the exposed areas but not in unexposed areas. These patterns showed clear image lines without webbing or meandering. ${ }^{20}$ Thus, the PVS molecule which contains the silylvinyl group as a side chain was found to act as negative working resist by exposing to a deep UV light.
Further details of the lithographic characteristics ${ }^{10,28,29}$ of PVS will be discussed elsewhere, ${ }^{30}$ including those of PVS exposed to ultraviolet light and an electron beam.

\section{CONCLUSIONS}

When anionic polymerization of VS was carried out at $-78^{\circ} \mathrm{C}$ for less than $3 \mathrm{~h}$, not a gel-like product but linear polymer was prepared. On comparing the NMR and IR spectra of VS with those of the resultant polymer, the styryl group of VS was found to be chemoselectively polymerized, and most of the silylvinyl group of VS was found to remain unreacted by the anionic polymerization. The produced-polymer was confirmed to be poly[(4vinylphenyl)dimethylvinylsilane] (PVS). To suppress the two side reactions related to the silylvinyl group during chain propagation, namely, addition of the styryl living end to the silylvinyl group of the side chain and abstraction of a labile proton of the silylmethyl group by the styryl living end, the optimum conditions were proposed in this study: polymerization temperature as $-78^{\circ} \mathrm{C}$, Cumyl Cs as initiator, $1: 2$ mixture of Ether/ 1,3-DX as solvent, VS polymerized for 30 minutes, and subsequent killing of the styryl living ends carried out as soon as possible. The four PVS samples having narrow MWD and controlled molecular weights were prepared under optimum conditions. The preliminary experimental results of the lithographic characterization show PVS to act as a negative working resist.

Acknowledgments. The authors thank Dr. A. Yamamoto, Specialty Chemicals Research Center, Shin-Etsu Chemical Co., Ltd., Niigata, Japan, for useful suggestions of preparation of the VS monomer. Thanks are due to Mr. S. Asaumi, Tokyo Ohka Kogyo Co., Ltd., Kanagawa, Japan, for assistance with the lithographic evaluation of deep UV light.

\section{REFERENCES}

1. K. Se, O. Watanabe, Y. Isono, and T. Fujimoto, Makromol. Chem., Makromol. Symp., 25, 249 (1989).

2. K. Se, H. Yamazaki, T. Shibamoto, A. Takano, and T. Fujimoto, Macromolecules, to be submitted.

3. M. Kijima, K. Se, and T. Fujimoto, Polymer, 33, 2402 (1992); ibid., 38, (1997), in press.

4. K. Se, M. Kijima, R. Ohtomo, and T. Fujimoto, J. Polym. Sci., Polym. Chem. Ed., 35 (1997), in press.

5. L. F. Thompson, C. G. Willson, and J. M. Frechet, in "Materials for Microlithography," ACS Symposium Series 266, The American Chemical Society, New York, N.Y., 1984.

6. L. F. Thompson, C. G. Willson, and S. Tagawa, in "Polymers 
for Microelectronics, Resists and Dielectrics," ACS Symposium Series 537, The American Chemical Society, Washington, D.C., 1994.

7. E. D. Feit and L. E. Stillwagon, Polym. Eng. Sci., 20, 1058 (1980).

8. T. Masuda, K. Kitagawa, T. Inoue, and S. Onogi, Macromolecules, 3, 116 (1970).

9. K. Itaya, K. Shibayama, and T. Fujimoto, J. Electrochem. Soc., 129, 663 (1982).

10. H. S. Choong and F. L. Kahn, J. Vac. Sci., Technol., 19, 1121 (1981).

11. K. Matsumura, K. Ueda, K. Se, and T. Fujimoto, Polym. Prepr. Jpn., 33, 153 (1984).

12. A. Takano, M. Okada, T. Nose, and T. Fujimoto, Macromolecules, 25, 3596 (1992).

13. R. Asami, J. Oku, M. Takeuchi, K. Nakamura, and M. Takaki, Polym. J., 20, 699 (1988).

14. J. Oku, M. Takeuchi, A. Saito, and R. Asami, Polym. J., 24, 1409 (1992).

15. S. Imamura, T. Tanaka, K. Harada, and S. Sugawara, J. Appl. Polym. Sci., 27, 937 (1982).

16. M. Bowden, J. Solid-State Technol., 24, 73 (1981).

17. B. Lin, J. Solid-State Technol., 26, 105 (1983).

18. T. Fujimoto, K. Tani, K. Takano, M. Ogawa, and M. Nagasawa, Macromolecules, 11, 678 (1983).
19. H. Funabashi, H. Miyamoto, Y. Isono, T. Fujimoto, Y. Matsushita, and M. Nagasawa, Macromolecules, 16, 1 (1983).

20. K. Se, M. Kijima, and T. Fujimoto, Polym. J., 20, 791 (1988)

21. R. Asami, M. Takaki, and H. Hanahara, Macromolecules, 16, $628(1983)$

22. K. Se, M. Suzuki, T. Matsuo, and T. Ueno, Kobunshi Ronbunshu, 49, 817 (1992)

23. Unpublished data determined by the present authors.

24. M. Izumi, K. Ogawa, S. Katou, J. Shiokawa, and T. Shiba, Ed., “Kiki Bunnseki no Tebiki," 2nd ed, Kagakudojin, Tokyo, 1996.

25. S. Nonogaki, H. Morishita, and N. Saitou, Appl. Polym. Symp., 23, 117 (1974)

26. K. Itaya, T. Fujimoto, M. Todoku, M Fukuda, and K. Kihara, Semiconductor and Integrated Circuit Prepr. Jpn., 23, 24 (1982).

27. M. Endo, Y. Tani, M. Sasago, and N. Nomura, J. Electrochem. Sci., 136, 2615 (1989).

28. R. Sezi, R. Leuschner, M. Sebald, H. Ahne, S. Birkle, and H. Borndorfer, Microelectric Eng., 11, 535 (1990).

29. A. Tamura and M. Sato, Proccedings, The Society of PhotoOptical Instrumentation Engineers (SPIE), Vol. 1465, The Society of Photo-Optical Instrumentation Engineers (SPIE), Washington, U.S.A., 1991, p. 35.

30. K. Se, K. Matsumura, T. Kazama, and T. Fujimoto, Polym. J., to be submitted. 